\title{
Diseño y validación de un cuestionario para estudiar la formación de entrenadores de fútbol base Design and validation of a questionnaire to assess football initiation coaches' training Paulo Paixão, Manuel Tomás Abad Robles, Francisco Javier Giménez Fuentes-Guerra *Instituto Politécnico de Beja (Portugal), **Universiad de Huelva (España)
}

Resumen. Con el objetivo de analizar la formación de los entrenadores de fútbol base en los clubes de la Asociación de Fútbol de Beja (Portugal) se diseñó un cuestionario de 117 items agrupados en ocho dimensiones (Características Sociodemográficas, Experiencia Deportiva, Aspectos Personales, Formación como Entrenador, Formación Continua, Concepción de Enseñanza, Planificación y Programación y Necesidades de Formación). Se llevó a cabo un análisis cualitativo y cuantitativo mediante la valoración de 12 expertos. Se analizó la validez de contenido a través del coeficiente V de Aiken, estableciendo un intervalo de confianza del 99 \%, y el coeficiente de variación. Para establecer la fiabilidad se analizó la consistencia interna por el método del Alfa de Cronbach (0.915) y la fiabilidad test-retest utilizando el coeficiente de correlación intraclase, 0.890 ( $p<0.01$ ). Los resultados demuestran que el cuestionario reúne suficientes propiedades psicométricas como para ser considerado una herramienta válida y fiable para estudiar y analizar las necesidades formativas de los entrenadores de fútbol de formación.

Palabras claves: validez, fiabilidad, técnico deportivo, fútbol de formación.

Abstract. IA questionnaire of 117 items grouped into eight dimensions (Socio-Demographic Characteristics, Sports Experience, Personal Aspects, Formation as Coach, Continuous Formation, Concepción Teaching, Planning and Programming and Needs of Formation) was designed in order to analyze the training of football coaches forming young players in clubs of the Football Association of Beja (Portugal). A qualitative and quantitative analysis was supported by 12 experts' evaluation. Content validity was analyzed through the V Aiken coefficient with a 99\% confidence interval, as well as through the coefficient of variation. Internal consistency was analyzed with the Cronbach's Alpha method (0.915) to establish the tool reliability; test-retest reliability was assessed through the intraclass correlation coefficient (ICC $=0.890$; $\mathrm{p}<0.01$ ). The results showed that the questionnaire gathers enough psychometric properties to be considered a valid and reliable tool to study and analyze football initiation coaches' training needs.

Key words: validity, reliability, sports technician, youth football.

\section{Introducción}

El nivel de formación y preparación metodológica del entrenador es un factor muy importante en el proceso de formación de las diferentes capacidades de los jugadores de fútbol, y puede determinar el éxito de los jóvenes futbolistas (Abad, Giménez, Robles \& Castillo, 2013; Cunha et al., 2010; Pulido et al., 2016; Stoszkowski \& Collins, 2017). Por eso, el papel del entrenador se asume como determinante para la calidad del entrenamiento durante las etapas de formación deportiva (Azpillaga, González, Irazusta \& Arruza, 2012; Lledó, Martínez \& Huertas, 2014). Este entrenador debe cumplir una serie de requisitos previos metodológicos en el proceso de enseñanza-entrenamiento (Bettega et al., 2018), morales y personales que le permitan influir positivamente en el proceso de capacitación (Taylor, Piper \& Garratt, 2016). Sus intervenciones tienden a definir los valores humanos y sociales, y por eso el entrenador es el agente primordial en la formación de los jóvenes (Light \& Harvey, 2017). Por todas estas razones hay necesidad de buscar y comprender mejor el perfil de los entrenadores que diariamente desarrollan su trabajo con los niños y jóvenes, y entender las carencias que persisten en este proceso (Giménez, Abad \& Robles, 2010; Mesquita et al., 2010). Según Abad, Giménez, Robles y Rodríguez (2011), las investigaciones sobre el entrenador de fútbol base son escasas, por lo que se hace necesaria la realización de estudios al respecto. Esta escasez de investigaciones también se verifica en Portugal (Cunha et al., 2010), contexto donde se pretende desarrollar la investigación.

Para que consigamos registrar una imagen verdaderamente precisa del entrenador, será necesario desarrollar una herramienta de observación específica para este deporte, y validarla (Brewer \& Jones, 2002). Consideramos importante y necesario desarrollar un instrumento válido, fiable y vinculado al contexto para así registrar la actuación del entrenador (Brewer \& Jones, 2002). Basándonos en Thomas y Nelson (2007), consideramos que el cuestionario es una excelente herramienta para investigar sobre el entrenador de fútbol base. Sin embargo, Ortega, Calderón, Palao, y Puigcerver (2008) afirman que en muchas ocasiones el cuestionario no registra realmente aquello que pretende medir, debido

Fecha recepción: 12-02-18. Fecha de aceptación: 31-10-18 Fecha recepción: 1
Paulo Paixão

paulo.paixao@ipbeja.pt a una escasa rigurosidad científica, apuntando tanto al diseño, como principalmente a su validación. A pesar de ser un instrumento muy utilizado, son muy pocos los cuestionarios que se diseñan y validan siguiendo los procesos metodológicos adecuados (Burgos, 2006). Butragueño y Benito (2014) constatan que muchos expertos recomiendan el uso de instrumentos de medición válidos y fiables, pero muy pocos estudios han discutido la validación de dichos instrumentos.

Por este motivo, el objetivo de este trabajo ha sido el diseño y validación de un cuestionario a través de un análisis cuantitativo y cualitativo (Ortega et al., 2018; García-Martín, Antúnez \& Ibáñez, 2016; Yagüe, Herrero, Tabernero \& Veroz, 2017), con el fin de obtener una herramienta válida y fiable que nos informe sobre la formación de los entrenadores de los clubes de la Asociación de Fútbol de Beja (Portugal). Los datos que serán recogidos con esta herramienta ayudarán a percibir mejor el proceso de formación de los entrenadores y su entrenamiento con los jóvenes futbolistas y, también nos ayudarán a hacer propuestas de formación futuras reales y útiles.

\section{Material y Método}

El presente trabajo responde a un diseño de investigación mixto, concretamente denominado triangulación de datos, donde buscamos realizar una evaluación cuantitativa y cualitativa (Östlund, Kidd, Wengström \& Rowa-Dewar, 2011), ya que partimos de diferentes fuentes de datos con la finalidad de confrontar informaciones complementarias (Castañer, Camerino \& Anguera, 2013).

\section{Participantes}

Para la validación de contenido se contó con un grupo homogéneo de 12 jueces-expertos (Robles, Robles, Giménez \& Abad, 2016; Maldonado, Pacheco \& Zamarripa, 2017; Yagüe, Herrero, Tabernero \& Veroz, 2017).A continuación, se detalla brevemente los perfiles de cada uno de ellos (Tabla 1).

\section{Instrumento}

Para el diseño del cuestionario nos basamos en el trabajo deAbad, Benito, Giménez y Robles (2011). A partir de este estudio y, teniendo en cuenta las características del fútbol en Portugal, se seleccionaron ocho dimensiones: «Características Sociodemográficas», «Experiencia Deportiva», «Aspectos Personales», «Formación como Entrenador», 


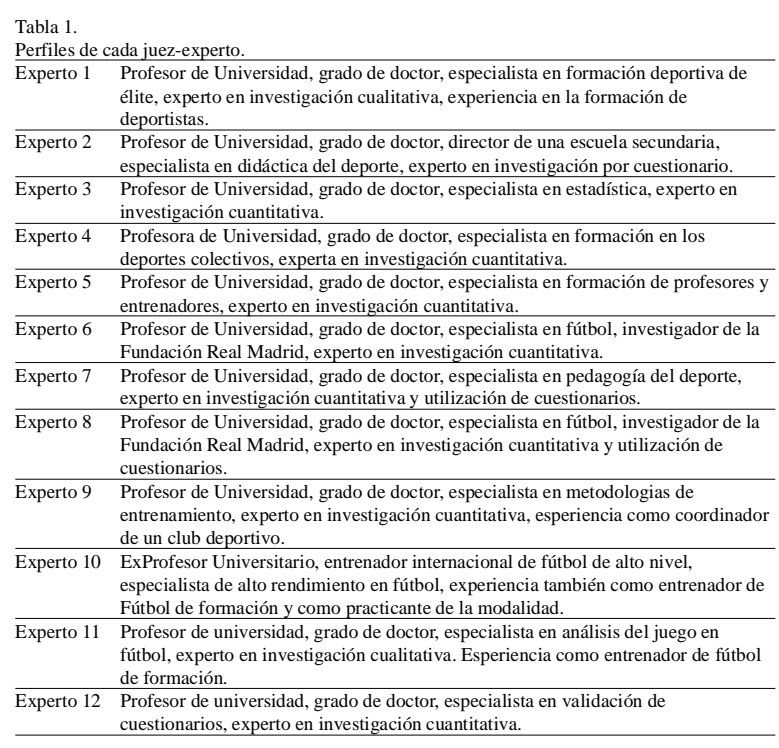

«Formación Continua», «Concepción de enseñanza», «Planificación y Programación» y «Necesidades de Formación». En el siguiente paso, los investigadores seleccionaron aquellas preguntas que consideraron oportunas, adaptándolas al contexto en el que se encuadra el presente estudio (Brewer \& Jones, 2002), eliminando las que se consideraron menos útiles y añadiendo nuevas preguntas. Para no dar lugar a una falta de comprensión o de entendimiento por parte de los entrenadores, se decidió redactar todas las preguntas de manera neutra, es decir, no se redactaron de forma positiva ni de forma negativa (Robles et al., 2016).

\section{Procedimiento}

Una vez elaborado el cuestionario preliminar, se procedió a la validación del mismo. Como se trata de evaluar a los entrenadores, se consideró el modelo de validación propuesto por Brewer y Jones (2002). También tuvimos en cuenta todas las recomendaciones de las fases de validación usadas por Butragueño y Benito (2014), pero adoptamos una validación centrada en tres fases (Simón, Fernández \& Contreras, 2017) distintas y concretas utilizando el método Delphi (Saldaña \& García, 2013; Otero, González \& Calvo, 2012). En la primera fase de validación, se pretendió hacer una evaluación general del cuestionario con el fin demejorar e identificar los vacíos en el instrumento de recogida de datos (Nuviala, Tamayo, Iranzo \& Falcón, 2008). A fin de facilitar esta tarea, se presentó un protocolo para la evaluación general del cuestionario que analizaba su presentación, las instrucciones para respuesta, la dificultad de respuesta, el orden de las preguntas y la extensión del cuestionario (Sierra, 2001). En una segunda fase también se han evaluado de forma general las dimensiones del cuestionario en cuanto a su pertinencia, redacción, claridad, comprensión y plenitud(Sierra, 2001). En la tercera fase de validación se diseñó una hoja de registro que se pasaría a los mismos jueces-expertos (Robles et al., 2016), en la cual se les pedía que valoraran mediante una escala Likert de tipo ordinal (Maldonado et al., 2017), en un rango de uno a seis. Se utilizó esta escala para evitar la tendencia a poner las respuestas en el valor central (Pereira, 2008), siendo el uno la puntuación más baja posible (nada adecuada) y el seis la puntuación más alta posible (muy adecuada). Se valoraron la pertinencia, el contenido y la redacción, de cada una de las preguntas del cuestionario (Butragueño \& Benito, 2014). Además, se les proponía la oportunidad de realizar cualquier sugerencia a las preguntas (AlmonacidFierro, Feu \& Vizuete, 2018), aportando cuestiones alternativas a las ya elaboradas o añadiendo cualquier otra que considerara oportuna.

\section{Validez y fiabilidad}

Para determinar la validez de contenido del cuestionario y con el fin de objetivar los resultados se procedió a realizar un análisis cuantitativo y otro cualitativo (Ortega et al., 2018) a partir de los datos obtenidos en las hojas de registros aportadas por los jueces-expertos (Latorre \& Pantoja, 2012). Finalmente, para el análisis de la fiabilidad se aplicaron los cuestionarios a una muestra piloto de 20 entrenadores del distrito Beja - Portugal, escogidos al azar (Ortega et al., 2018), lo que permitió contextualizar el instrumento al entorno específico (Brewer \& Jones, 2002). Con el objetivo de llevar a cabo el análisis de fiabilidad test-retest (Nevil, Lane, Kilgour, Bowes \& Whyte, 2001), cada entrenador fue sometido al cuestionario en dos ocasiones con un intervalo de tiempo, entre cada aplicación, de tres semanas para evitar cualquier posible efecto adverso de memoria (Lago, 2009). Los encuestados fueron informados sobre el objetivo del estudio y se les garantizó la confidencialidad de la información recogida bajo la directiva comunitaria 95/46/CE, relativa a la protección y tratamiento de los datos personales.

\section{Análisis estadístico}

En relación con el análisis cuantitativo, se calcularon la media, la desviación típica y el coeficiente de variación de cada pregunta, a través del programa SPSS (Statistical Package for Social Sciences) versión 23.0 para Windows. Además, se halló el coeficiente de validez de contenido $V$ de Aiken (Aiken, 1985), dicho coeficiente se calculó mediante la aplicación informática diseñada por Merino \& Livia (2009), la cual permite obtener los intervalos de confianza, a través del método score (Peinfield \& Giacobbi, 2004). Para calculcular el valor crítico exacto de la V de Aiken se usó la fórmula propuesta por Aiken (1985) para grandes muestras. Este valor indica que ítems son aceptados, siempre y cuando superen el valor crítico indicado de la V de Aiken. Para ello se tien en cuenta la fórmula:

$$
V=\frac{z}{0.2 \sqrt{\frac{3 m n(\mathrm{c}-1)}{(\mathrm{c}+1)}}}+0.5
$$

Donde $z$ es el nivel de significación, $m$ el número de ítems que los expertos tienen que valorar, $n$ es el número de jueces expertos que participan en el estudio y c el valor máximo que puede valorar un ítem.

Respecto al análisis cualitativo, se tomaron en cuenta todas las aportaciones de los distintos jueces-expertos en cada una de las preguntas. A partir de los resultados obtenidos en ambos análisis se establecieron los criterios de exclusión o revisión para cada ítem utilizados por Robles et al. (2016).

\section{Criterios de exclusión:}

Primer criterio: que la pregunta obtenga una Vde Aiken inferior a lo establecido en el intervalo de confianza (99\%) o no supere el valor crítico establecido ( $V=0,71$, y posea un coeficiente de variación superior a un $25 \%$ en los parámetros de pertinencia y contenido evaluados por los expertos. Segundo criterio: que al menos dos jueces-expertos soliciten la exclusión de la pregunta. Tercer criterio: que un juez-experto solicite la exclusión de una pregunta y el grupo de investigación lo estime oportuno.

\section{Criterios de revisión:}

Primer criterio de revisión: que la pregunta obtenga una $V$ de Aiken inferior al valor crítico establecido 0.71 o posea un coeficiente de variación superior al 20\% en el parámetro de redacción evaluado por los expertos. Segundo criterio de revisión: que lo indique al menos uno de los jueces-expertos. Mediante la aplicación de estos criterios, sobre el cuestionario preliminar, se obtuvo el cuestionario definitivo.

Para el análisis estadístico de fiabilidad del cuestionario definitivo, se utilizó el coeficiente Alpha de Cronbach para evaluar la consistencia interna (Garrido, Zagalaz, Torres \& Romero, 2010; Checa \& Bohórquez, 2018). La fiabilidad test-retest se analizó mediante el coeficiente de correlación intraclase, que mide el nivel de concordancia entre dos medidas y nos indica si el resultado de la media tiene estabilidad temporal (Butragueño \& Benito, 2014). 


\section{Resultados}

\section{Análisis cuantitativo general}

Teniendo en cuenta una primera evaluación global del cuestionario, no sometiendo a los criterios de exclusión y revisión los aspectos generales como presentación, instrucciones, dificultad, orden y extensión (Sierra, 2001), se observó que en el coeficiente Vde Aiken, la extensión fue el único aspecto con una $V$ de Aiken inferior al valor crítico establecido 0.71 , por lo que se revisó esta cuestión. Sin embargo, no presentó un coeficiente de variación superior a un $25 \%$ en los distintos parámetros.

Observando cada dimensión del cuestionario en particular y atendiendo al primer criterio de exclusión hay que señalar que ninguna dimensión fue eliminada, ya que todas obtuvieron valores en el coeficiente $V$ de Aiken dentro de lo establecido en el intervalo de confianza (99\%), además de no poseer un coeficiente de variación superior a un 25\% en ninguno de los cinco parámetros evaluados por los expertos: pertinencia, redacción, claridad, comprensión y plenitud(Sierra, 2001).

\section{Análisis cuantitativo específico}

Contemplando cada pregunta de forma específica y atendiendo al primer criterio de exclusión se verifica queninguna pregunta fue eliminada, ya que todas obtuvieron valores en el coeficiente $V$ de Aiken dentro de lo establecido en el intervalo de confianza (99\%) y, además de no poseer un coeficiente de variación superior a un $25 \%$ en los parámetros de pertinencia y contenido evaluados por los expertos. Podemos destacar que las preguntas 43 y 44 se encuentran en un límite aceptable con 25.85\% (Tabla 2).

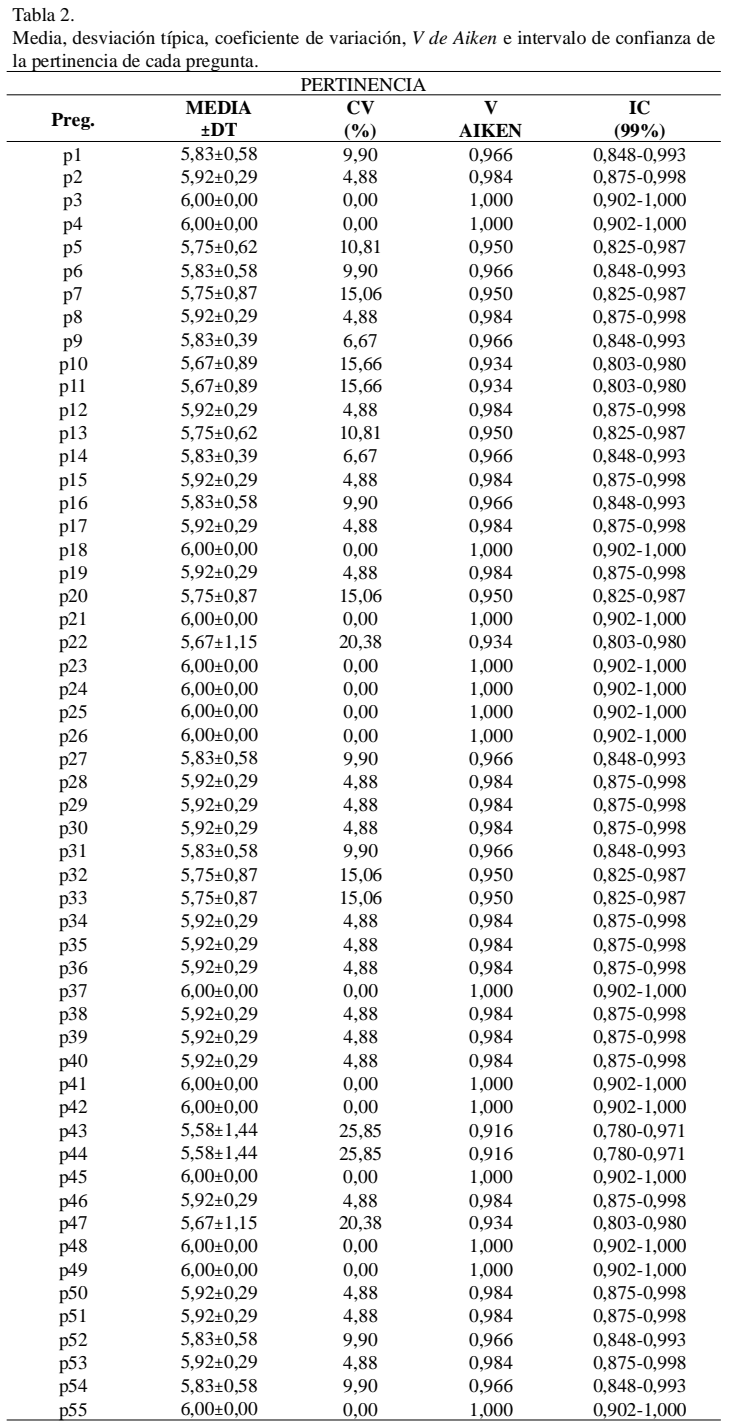

Considerando el parámetro de la pertinencia (Tabla 2), en relación con la revisión de las preguntas y teniendo en cuenta el primer criterio, se destaca que a pesar de que se obtuvieron valores del coeficiente $V d e$ Aiken por encima del valor crítico establecido 0.71 , algunas de las preguntas presentaron un coeficiente de variación por encima del $20 \%$, por lo que pasaron a revisión un total de cuatro preguntas: las preguntas 22, 43 y 44 de la dimensión formación como entrenador y, la pregunta 47 de la dimensión formación continua.

Teniendo en cuenta el parámetro del contenido (Tabla 3), en relación con la revisión de las preguntas y atendiendo al primer criterio, hay destacar que a pesar de que se obtuvieron valores del coeficiente $V$ de Aiken por encima del valor crítico establecido 0.71 , algunas de las preguntas presentaron un coeficiente de variación por encima del $20 \%$, por lo que pasaron a revisión un total de cinco preguntas: las preguntas 43 y 44 de la dimensión formación como entrenador, las cuestiones 47 y 48 de la dimensión formación continua y la pregunta 85 de la dimensión necesidades de formación.

Considerando el parámetro de redacción (Tabla 4), en relación con la revisión de las preguntas y teniendo en cuenta el primer criterio, hay que señalar que a pesar de que se obtuvieron valores del coeficiente $V d e$ Aiken por encima del valor crítico estableciso 0.71 , algunos de los ítems presentaron un coeficiente de variación por encima del $20 \%$, por lo que pasaron a revisión un total de nueve cuestiones: las preguntas 47 y 48 de la dimensión formación continua, las cuestiones 66, 67, 72, 83 y 84 de la dimensión planificación y programación, y los ítems 85 y 106 de la dimensión necesidades de formación.

\begin{tabular}{|c|c|c|c|c|}
\hline p56 & $5,75 \pm, 87$ & 15,06 & 0,950 & $0,825-0,987$ \\
\hline p57 & $6,00 \pm, 00$ & 0,00 & 1,000 & $0,902-1,000$ \\
\hline p58 & $6,00 \pm, 00$ & 0,00 & 1,000 & $0,902-1,000$ \\
\hline p59 & $6,00 \pm 0,00$ & 0,00 & 1,000 & $0,902-1,000$ \\
\hline p60 & $5,83 \pm, 58$ & 9,90 & 0,966 & $0,848-0,993$ \\
\hline p61 & $5,83 \pm, 58$ & 9,90 & 0,966 & $0,848-0,993$ \\
\hline $\mathrm{p} 62$ & $6,00 \pm, 00$ & 0,00 & 1,000 & $0,902-1,000$ \\
\hline p63 & $5,92 \pm, 29$ & 4,88 & 0,984 & $0,875-0,998$ \\
\hline p64 & $5,92 \pm, 29$ & 4,88 & 0,984 & $0,875-0,998$ \\
\hline p65 & $6,00 \pm 0,00$ & 0,00 & 1,000 & $0,902-1,000$ \\
\hline p66 & $6,00 \pm, 00$ & 0,00 & 1,000 & $0,902-1,000$ \\
\hline $\mathrm{p} 67$ & $6,00 \pm, 00$ & 0,00 & 1,000 & $0,902-1,000$ \\
\hline p68 & $6,00 \pm, 00$ & 0,00 & 1,000 & $0,902-1,000$ \\
\hline p69 & $6,00 \pm, 00$ & 0,00 & 1,000 & $0,902-1,000$ \\
\hline p70 & $6,00 \pm, 00$ & 0,00 & 1,000 & $0,902-1,000$ \\
\hline p71 & $6,00 \pm, 00$ & 0,00 & 1,000 & $0,902-1,000$ \\
\hline p72 & $5,92 \pm, 29$ & 4,88 & 0,984 & $0,875-0,998$ \\
\hline p73 & $6,00 \pm 0,00$ & 0,00 & 1,000 & $0,902-1,000$ \\
\hline p74 & $5,92 \pm, 29$ & 4,88 & 0,984 & $0,875-0,998$ \\
\hline p75 & $5,92 \pm, 29$ & 4,88 & 0,984 & $0,875-0,998$ \\
\hline p76 & $6,00 \pm, 00$ & 0,00 & 1,000 & $0,902-1,000$ \\
\hline p77 & $5,92 \pm, 29$ & 4,88 & 0,984 & $0,875-0,998$ \\
\hline p78 & $5,92 \pm, 29$ & 4,88 & 0,984 & $0,875-0,998$ \\
\hline p79 & $5,92 \pm, 29$ & 4,88 & 0,984 & $0,875-0,998$ \\
\hline p80 & $6,00 \pm 0,00$ & 0,00 & 1,000 & $0,902-1,000$ \\
\hline p81 & $6,00 \pm, 00$ & 0,00 & 1,000 & $0,902-1,000$ \\
\hline p82 & $5,92 \pm 0,29$ & 4,88 & 0,984 & $0,875-0,998$ \\
\hline p83 & $6,00 \pm, 00$ & 0,00 & 1,000 & $0,902-1,000$ \\
\hline p84 & $5,83 \pm, 58$ & 9,90 & 0,966 & $0,848-0,993$ \\
\hline p85 & $6,00 \pm, 00$ & 0,00 & 1,000 & $0,902-1,000$ \\
\hline p86 & $6,00 \pm, 00$ & 0,00 & 1,000 & $0,902-1,000$ \\
\hline p87 & $5,92 \pm, 29$ & 4,88 & 0,984 & $0,875-0,998$ \\
\hline p88 & $6,00 \pm, 00$ & 0,00 & 1,000 & $0,902-1,000$ \\
\hline p89 & $6,00 \pm, 00$ & 0,00 & 1,000 & $0,902-1,000$ \\
\hline p90 & $5,92 \pm, 29$ & 4,88 & 0,984 & $0,875-0,998$ \\
\hline p91 & $5,92 \pm, 29$ & 4,88 & 0,984 & $0,875-0,998$ \\
\hline p92 & $5,83 \pm, 58$ & 9,90 & 0,966 & $0,848-0,993$ \\
\hline p93 & $6,00 \pm, 00$ & 0,00 & 1,000 & $0,902-1,000$ \\
\hline p94 & $6,00 \pm, 00$ & 0,00 & 1,000 & $0,902-1,000$ \\
\hline p95 & $6,00 \pm, 00$ & 0,00 & 1,000 & $0,902-1,000$ \\
\hline p96 & $6,00 \pm, 00$ & 0,00 & 1,000 & $0,902-1,000$ \\
\hline p97 & $6,00 \pm, 00$ & 0,00 & 1,000 & $0,902-1,000$ \\
\hline p98 & $5,92 \pm, 29$ & 4,88 & 0,984 & $0,875-0,998$ \\
\hline p99 & $5,92 \pm, 29$ & 4,88 & 0,984 & $0,875-0,998$ \\
\hline $\mathrm{p} 100$ & $6,00 \pm 0,00$ & 0,00 & 1,000 & $0,902-1,000$ \\
\hline p101 & $6,00 \pm, 00$ & 0,00 & 1,000 & $0,902-1,000$ \\
\hline p102 & $5,92 \pm, 29$ & 4,88 & 0,984 & $0,875-0,998$ \\
\hline p103 & $6,00 \pm, 00$ & 0,00 & 1,000 & $0,902-1,000$ \\
\hline p104 & $6,00 \pm, 00$ & 0,00 & 1,000 & $0,902-1,000$ \\
\hline p105 & $6,00 \pm, 00$ & 0,00 & 1,000 & $0,902-1,000$ \\
\hline p106 & $5,92 \pm, 29$ & 4,88 & 0,984 & $0,875-0,998$ \\
\hline p107 & $6,00 \pm 0,00$ & 0,00 & 1,000 & $0,902-1,000$ \\
\hline p108 & $6,00 \pm, 00$ & 0,00 & 1,000 & $0,902-1,000$ \\
\hline p109 & $5,92 \pm, 29$ & 4,88 & 0,984 & $0,875-0,998$ \\
\hline p110 & $5,92 \pm, 29$ & 4,88 & 0,984 & $0,875-0,998$ \\
\hline p111 & $5,83 \pm, 58$ & 9,90 & 0,966 & $0,848-0,993$ \\
\hline p112 & $6,00 \pm, 00$ & 0,00 & 1,000 & $0,902-1,000$ \\
\hline p113 & $5,92 \pm, 29$ & 4,88 & 0,984 & $0,875-0,998$ \\
\hline p114 & $5,92 \pm, 29$ & 4,88 & 0,984 & $0,875-0,998$ \\
\hline p115 & $5,92 \pm, 29$ & 4,88 & 0,984 & $0,875-0,998$ \\
\hline p116 & $6,00 \pm, 00$ & 0,00 & 1,000 & $0,902-1,000$ \\
\hline p117 & $5,75 \pm, 87$ & 15,06 & 0,950 & $0,825-0,987$ \\
\hline
\end{tabular}


Tabla 3.

Media, desviación típica, coeficiente de variación, $V$ de Aiken e intervalo de confianza del contenido de cada pregunta.

\begin{tabular}{|c|c|c|c|c|}
\hline \multirow[b]{2}{*}{ Preg. } & MEDIA & & & \multirow{2}{*}{$\begin{array}{c}\text { IC } \\
(99 \%)\end{array}$} \\
\hline & $\begin{array}{l}\text { MEDDA } \\
\pm \text { DT }\end{array}$ & (\%) & V AIKEN & \\
\hline $\mathrm{p} 1$ & $6,00 \pm 0,00$ & 0,00 & 1,000 & $0,902-1,000$ \\
\hline p2 & $6,00 \pm 0,00$ & 0,00 & 1,000 & $0,902-1,000$ \\
\hline p3 & $5,67 \pm 0,89$ & 15,66 & 0,934 & $0,803-0,980$ \\
\hline $\mathrm{p} 4$ & $6,00 \pm 0,00$ & 0,00 & 1,000 & $0,902-1,000$ \\
\hline p5 & $5,83 \pm 0,58$ & 9,90 & 0,966 & $0,848-0,993$ \\
\hline p6 & $5,83 \pm 0,39$ & 6,67 & 0,966 & $0,848-0,993$ \\
\hline p7 & $5,83 \pm 0,39$ & 6,67 & 0,966 & $0,848-0,993$ \\
\hline p8 & $5,75 \pm 0,45$ & 7,87 & 0,950 & $0,825-0,987$ \\
\hline p9 & $5,83 \pm 0,39$ & 6,67 & 0,966 & $0,848-0,993$ \\
\hline p10 & $5,75 \pm 0,62$ & 10,81 & 0,950 & $0,825-0,987$ \\
\hline p11 & $5,67 \pm 0,89$ & 15,66 & 0,934 & $0,803-0,980$ \\
\hline p12 & $6,00 \pm 0,00$ & 0,00 & 1,000 & $0,902-1,000$ \\
\hline p13 & $6,00 \pm 0,00$ & 0,00 & 1,000 & $0,902-1,000$ \\
\hline p14 & $6,00 \pm 0,00$ & 0,00 & 1,000 & $0,902-1,000$ \\
\hline p15 & $6,00 \pm 0,00$ & 0,00 & 1,000 & $0,902-1,000$ \\
\hline p16 & $6,00 \pm 0,00$ & 0,00 & 1,000 & $0,902-1,000$ \\
\hline p17 & $5,92 \pm 0,29$ & 4,88 & 0,984 & $0,875-0,998$ \\
\hline p18 & $5,92 \pm 0,29$ & 4,88 & 0,984 & $0,875-0,998$ \\
\hline p19 & $5,92 \pm 0,29$ & 4,88 & 0,984 & $0,875-0,998$ \\
\hline p20 & $5,92 \pm 0,29$ & 4,88 & 0,984 & $0,875-0,998$ \\
\hline p21 & $5,92 \pm 0,29$ & 4,88 & 0,984 & $0,875-0,998$ \\
\hline p22 & $5,92 \pm 0,29$ & 4,88 & 0,984 & $0,875-0,998$ \\
\hline $\mathrm{p} 23$ & $5,83 \pm 0,58$ & 9,90 & 0,966 & $0,848-0,993$ \\
\hline p 24 & $5,92 \pm 0,29$ & 4,88 & 0,984 & $0,875-0,998$ \\
\hline p25 & $5,92 \pm 0,29$ & 4,88 & 0,984 & $0,875-0,998$ \\
\hline p26 & $5,92 \pm 0,29$ & 4,88 & 0,984 & $0,875-0,998$ \\
\hline p27 & $5,92 \pm 0,29$ & 4,88 & 0,984 & $0,875-0,998$ \\
\hline p28 & $5,92 \pm 0,29$ & 4,88 & 0,984 & $0,875-0,998$ \\
\hline p29 & $5,92 \pm 0,29$ & 4,88 & 0,984 & $0,875-0,998$ \\
\hline $\mathrm{p} 30$ & $5,92 \pm 0,29$ & 4,88 & 0,984 & $0,875-0,998$ \\
\hline $\mathrm{p} 31$ & $5,83 \pm 0,58$ & 9,90 & 0,966 & $0,848-0,993$ \\
\hline $\mathrm{p} 32$ & $5,92 \pm 0,29$ & 4,88 & 0,984 & $0,875-0,998$ \\
\hline p33 & $5,92 \pm 0,29$ & 4,88 & 0,984 & $0,875-0,998$ \\
\hline $\mathrm{p} 34$ & $5,92 \pm 0,29$ & 4,88 & 0,984 & $0,875-0,998$ \\
\hline p35 & $5,92 \pm 0,29$ & 4,88 & 0,984 & $0,875-0,998$ \\
\hline p36 & $5,92 \pm 0,29$ & 4,88 & 0,984 & $0,875-0,998$ \\
\hline p37 & $5,92 \pm 0,29$ & 4,88 & 0,984 & $0,875-0,998$ \\
\hline p38 & $5,83 \pm 0,58$ & 9,90 & 0,966 & $0,848-0,993$ \\
\hline p39 & $5,92 \pm 0,29$ & 4,88 & 0,984 & $0,875-0,998$ \\
\hline $\mathrm{p} 40$ & $5,83 \pm 0,58$ & 9,90 & 0,966 & $0,848-0,993$ \\
\hline $\mathrm{p} 41$ & $6,00 \pm 0,00$ & 0,00 & 1,000 & $0,902-1,000$ \\
\hline $\mathrm{p} 42$ & $6,00 \pm 0,00$ & 0,00 & 1,000 & $0,902-1,000$ \\
\hline $\mathrm{p} 43$ & $5,58 \pm 1,44$ & 25,85 & $\begin{array}{l}0,916 \\
0,900\end{array}$ & $0,780-0,971$ \\
\hline $\mathrm{p} 44$ & $5,58 \pm 1,44$ & 25,85 & 0,916 & $0,780-0,971$ \\
\hline p45 & $6,00 \pm 0,00$ & 0,00 & 1,000 & $0,902-1,000$ \\
\hline p46 & $5,83 \pm 0,58$ & 9,90 & 0,966 & $0,848-0,993$ \\
\hline p47 & $5,67 \pm 1,15$ & 20,38 & 0,934 & $0,803-0,980$ \\
\hline p48 & $5,58 \pm 1,16$ & 20,86 & 0,900 & $0,759-0,963$ \\
\hline p49 & $6,00 \pm 0,00$ & 0,00 & 1,000 & $0,902-1,000$ \\
\hline $\mathrm{p} 50$ & $5,83 \pm 0,39$ & 6,67 & 0,966 & $0,848-0,993$ \\
\hline p51 & $5,83 \pm 0,58$ & 9,90 & 0,966 & $0,848-0,993$ \\
\hline p52 & $5,92 \pm 0,29$ & 4,88 & 0,984 & $0,875-0,998$ \\
\hline p53 & $5,92 \pm 0,29$ & 4,88 & 0,984 & $0,875-0,998$ \\
\hline p54 & $5,83 \pm 0,58$ & 9,90 & 0,966 & $0,848-0,993$ \\
\hline p55 & $6,00 \pm 0,00$ & 0,00 & 1,000 & $0,902-1,000$ \\
\hline
\end{tabular}

\section{Análisis cualitativo general}

Atendiendo al segundo y tercer criterio de exclusión se observa que ninguna pregunta fue eliminada del cuestionario preliminar. Respecto a la revisión general del cuestionario y sus dimensiones, teniendo en cuenta el segundo criterio expuesto, se revisaron las dimensiones Experiencia Deportiva, Formación Continua y Necesidades de Formación. Las propuestas de modificaciones más importantes hacían referencia a contemplar otras maneras de formación continua y a señalar en el cuestionario las dimensiones del mismo.

\section{Análisis cualitativo específico}

Teniendo en cuenta el tercer criterio de exclusión, una de las opciones de respuesta de la pregunta tres de la dimensión Características Sociodemográficas fue retirada, ya que un juez-experto lo solicitó y el grupo de investigación lo estimó oportuno. Atendiendo al segundo criterio de revisión y, con las sugerencias de los jueces-expertos en la dimensión Experiencia Deportiva se modificaron las preguntas 6, 7, 9y 23. En la dimensión Aspectos Personales se modificó la pregunta ocho. En la dimensión Formación Continua se modificaron las preguntas 51 y 53. En la dimensión Concepción de Enseñanza se modificó la pregunta 59, retirando la palabra «enseñar», sustituyéndola por «transmitir valores». En la dimensión Planificación y Programación se cambiaron las preguntas 66, 67, 72, 83 y 84. En la dimensión Necesidades de Formación se reestructuraron las preguntas 85 y 86 . Basándonos en el tercer criterio de exclusión, las preguntas 43 y 44 de la dimensión Formación como Entrenador fueron retiradas, ya que un juez-experto lo solicitó y el grupo de investigación lo estimó oportuno. Esta dimensión se ha dividido en dos grupos: la formación general y la formación específica,

\begin{tabular}{|c|c|c|c|c|}
\hline p56 & $5,75 \pm 0,87$ & 15,06 & 0,950 & $0,825-0,987$ \\
\hline p57 & $6,00 \pm 0,00$ & 0,00 & 1,000 & $0,902-1,000$ \\
\hline p58 & $5,92 \pm 0,29$ & 4,88 & 0,984 & $0,875-0,998$ \\
\hline p59 & $5,92 \pm 0,29$ & 4,88 & 0,984 & $0,875-0,998$ \\
\hline p60 & $5,83 \pm 0,58$ & 9,90 & 0,966 & $0,848-0,993$ \\
\hline p61 & $5,83 \pm 0,58$ & 9,90 & 0,966 & $0,848-0,993$ \\
\hline p62 & $6,00 \pm 0,00$ & 0,00 & 1,000 & $0,902-1,000$ \\
\hline p63 & $5,50 \pm 1,00$ & 18,18 & 0,900 & $0,759-0,963$ \\
\hline p64 & $5,92 \pm 0,29$ & 4,88 & 0,984 & $0,875-0,998$ \\
\hline p65 & $6,00 \pm 0,00$ & 0,00 & 1,000 & $0,902-1,000$ \\
\hline p66 & $5,75 \pm 0,87$ & 15,06 & 0,950 & $0,825-0,987$ \\
\hline $\mathrm{p} 67$ & $5,75 \pm 0,87$ & 15,06 & 0,950 & $0,825-0,987$ \\
\hline p68 & $6,00 \pm 0,00$ & 0,00 & 1,000 & $0,902-1,000$ \\
\hline p69 & $6,00 \pm 0,00$ & 0,00 & 1,000 & $0,902-1,000$ \\
\hline p70 & $6,00 \pm 0,00$ & 0,00 & 1,000 & $0,902-1,000$ \\
\hline p71 & $6,00 \pm 0,00$ & 0,00 & 1,000 & $0,902-1,000$ \\
\hline p72 & $5,92 \pm 0,29$ & 4,88 & 0,984 & $0,875-0,998$ \\
\hline p73 & $6,00 \pm 0,00$ & 0,00 & 1,000 & $0,902-1,000$ \\
\hline p74 & $5,92 \pm 0,29$ & 4,88 & 0,984 & $0,875-0,998$ \\
\hline p75 & $5,92 \pm 0,29$ & 4,88 & 0,984 & $0,875-0,998$ \\
\hline p76 & $6,00 \pm 0,00$ & 0,00 & 1,000 & $0,902-1,000$ \\
\hline p77 & $5,92 \pm 0,29$ & 4,88 & 0,984 & $0,875-0,998$ \\
\hline p78 & $5,92 \pm 0,29$ & 4,88 & 0,984 & $0,875-0,998$ \\
\hline p79 & $5,92 \pm 0,29$ & 4,88 & 0,984 & $0,875-0,998$ \\
\hline p80 & $6,00 \pm 0,00$ & 0,00 & 1,000 & $0,902-1,000$ \\
\hline p81 & $6,00 \pm 0,00$ & 0,00 & 1,000 & $0,902-1,000$ \\
\hline p82 & $5,92 \pm 0,29$ & 4,88 & 0,984 & $0,875-0,998$ \\
\hline p83 & $6,00 \pm 0,00$ & 0,00 & 1,000 & $0,902-1,000$ \\
\hline p84 & $5,75 \pm 0,62$ & 10,81 & 0,950 & $0,825-0,987$ \\
\hline p85 & $5,67 \pm 1,15$ & 20,38 & 0,934 & $0,803-0,980$ \\
\hline p86 & $5,92 \pm 0,29$ & 4,88 & 0,984 & $0,875-0,998$ \\
\hline p87 & $5,92 \pm 0,29$ & 4,88 & 0,984 & $0,875-0,998$ \\
\hline p88 & $6,00 \pm 0,00$ & 0,00 & 1,000 & $0,902-1,000$ \\
\hline p89 & $6,00 \pm 0,00$ & 0,00 & 1,000 & $0,902-1,000$ \\
\hline p90 & $5,92 \pm 0,29$ & 4,88 & 0,984 & $0,875-0,998$ \\
\hline p91 & $5,92 \pm 0,29$ & 4,88 & 0,984 & $0,875-0,998$ \\
\hline p92 & $5,83 \pm 0,58$ & 9,90 & 0,966 & $0,848-0,993$ \\
\hline p93 & $6,00 \pm 0,00$ & 0,00 & 1,000 & $0,902-1,000$ \\
\hline p94 & $6,00 \pm 0,00$ & 0,00 & 1,000 & $0,902-1,000$ \\
\hline p95 & $6,00 \pm 0,00$ & 0,00 & 1,000 & $0,902-1,000$ \\
\hline p96 & $6,00 \pm 0,00$ & 0,00 & 1,000 & $0,902-1,000$ \\
\hline p97 & $6,00 \pm 0,00$ & 0,00 & 1,000 & $0,902-1,000$ \\
\hline p98 & $5,92 \pm 0,29$ & 4,88 & 0,984 & $0,875-0,998$ \\
\hline p99 & $5,92 \pm 0,29$ & 4,88 & 0,984 & $0,875-0,998$ \\
\hline p100 & $6,00 \pm 0,00$ & 0,00 & 1,000 & $0,902-1,000$ \\
\hline p101 & $6,00 \pm 0,00$ & 0,00 & 1,000 & $0,902-1,000$ \\
\hline p102 & $5,92 \pm 0,29$ & 4,88 & 0,984 & $0,875-0,998$ \\
\hline $\mathrm{p} 103$ & $6,00 \pm 0,00$ & 0,00 & 1,000 & $0,902-1,000$ \\
\hline p104 & $6,00 \pm 0,00$ & 0,00 & 1,000 & $0,902-1,000$ \\
\hline p105 & $6,00 \pm 0,00$ & 0,00 & 1,000 & $0,902-1,000$ \\
\hline p106 & $5,83 \pm 0,39$ & 6,67 & 0,966 & $0,848-0,993$ \\
\hline p107 & $6,00 \pm 0,00$ & 0,00 & 1,000 & $0,902-1,000$ \\
\hline p108 & $6,00 \pm 0,00$ & 0,00 & 1,000 & $0,902-1,000$ \\
\hline p109 & $5,92 \pm 0,29$ & 4,88 & 0,984 & $0,875-0,998$ \\
\hline p110 & $5,92 \pm 0,29$ & 4,88 & 0,984 & $0,875-0,998$ \\
\hline p111 & $5,83 \pm 0,58$ & 9,90 & 0,966 & $0,848-0,993$ \\
\hline p112 & $6,00 \pm 0,00$ & 0,00 & 1,000 & $0,902-1,000$ \\
\hline $\mathrm{p} 113$ & $5,92 \pm 0,29$ & 4,88 & 0,984 & $0,875-0,998$ \\
\hline p114 & $5,92 \pm 0,29$ & 4,88 & 0,984 & $0,875-0,998$ \\
\hline p115 & $5,92 \pm 0,29$ & 4,88 & 0,984 & $0,875-0,998$ \\
\hline p116 & $6,00 \pm 0,00$ & 0,00 & 1,000 & $0,902-1,000$ \\
\hline p117 & $5,75 \pm 0,87$ & 15,06 & 0,950 & $0,825-0,987$ \\
\hline
\end{tabular}

a partir de las sugerencias aportadas. Se añadieron también cinco nuevos items: promoción de valores deportivos, coaching deportivo, entrenamiento de porteros, scouting en el fútbol y didáctica del deporte. Se consideraron todas las sugerencias aportadas por los jueces expertos que se detallan en la Tabla 5.$$
\text { Tabla } 5 .
$$

\begin{tabular}{|c|c|c|}
\hline \multicolumn{3}{|c|}{ Exper } \\
\hline \multicolumn{3}{|c|}{ Características Sociodemográficas } \\
\hline P3 & Se podría retirar la opción doméstico. & E2 \\
\hline \multicolumn{3}{|c|}{ Experiencia Deportiva } \\
\hline P6 y 23 & Pondría otro rango de edad de 12-14 y después 15-18 años & E2 \\
\hline P7 & Puede ser que una persona esté dirigiendo a equipos de distintas categorías! & E1 \\
\hline P9 & $\begin{array}{c}\text { Creo que se puede poner: si contesta no en la pregunta nueve, pasa a la } \\
\text { pregunta.... }\end{array}$ & E4 \\
\hline \multicolumn{3}{|c|}{ Aspectos Personales } \\
\hline P8 & Pondría la categoría: otra & E2 \\
\hline \multicolumn{3}{|c|}{ Formación como Entrenador } \\
\hline Sugerencias & $\begin{array}{l}\text { Si es el elemento de formación general que los cursos tienen ahora no habría } \\
\text { técnico/táctica y otros. Debería reformular. }\end{array}$ & Éa E11 \\
\hline \multicolumn{3}{|c|}{ Formación Continua } \\
\hline P51 & $\begin{array}{l}\text { Propongo excluir a los monitores y mantener sólo los entrenadores, para no } \\
\text { crear confusión a los encuestados. }\end{array}$ & E11 \\
\hline P 53 & $\begin{array}{l}\text { No se accede al conocimiento, se accede a la información. Sugiero } \\
\text { reformulación. }\end{array}$ & E11 \\
\hline \multicolumn{3}{|c|}{ Concepción de enseñanza } \\
\hline $\mathrm{P} 59$ & retiraba "enseñar" y pondría transmitir valores (los valores se transmiten) & E2 \\
\hline \multicolumn{3}{|c|}{ Planificación y Programación } \\
\hline P66 & Sugiero: "Suelo programar el trabajo a implementar en ciclos mensuales." & E11 \\
\hline P67 & Sugiero: "Suelo programar el trabajo a implementar en cada semana." & E11 \\
\hline P72 & Sugiero: “Es fácil para mí para motivar a mis jugadores." & E11 \\
\hline P83 & $\begin{array}{l}\text { ¿Lo que es asistír "normalmente" a los entrenamientos? Sugiero } \\
\text { reformulación. }\end{array}$ & E11 \\
\hline P84 & Sugiero: "En el futuro, mi intención es entrenar a niveles superiores." & E11 \\
\hline \multicolumn{3}{|c|}{ Necesidades de Formación } \\
\hline P85 & Subdividir la cuestión en individual y colectiva. & E5 \\
\hline P86 & Sugiero reformular el término preparación física. & E2 \\
\hline (IE) & $\begin{array}{l}\text { ¿En qué contenidos tendría más interés en continuar su formación?: Aquí } \\
\text { añadiría un ítem: Promoción / reconocimiento de valores deportivos. }\end{array}$ & E1 \\
\hline
\end{tabular}




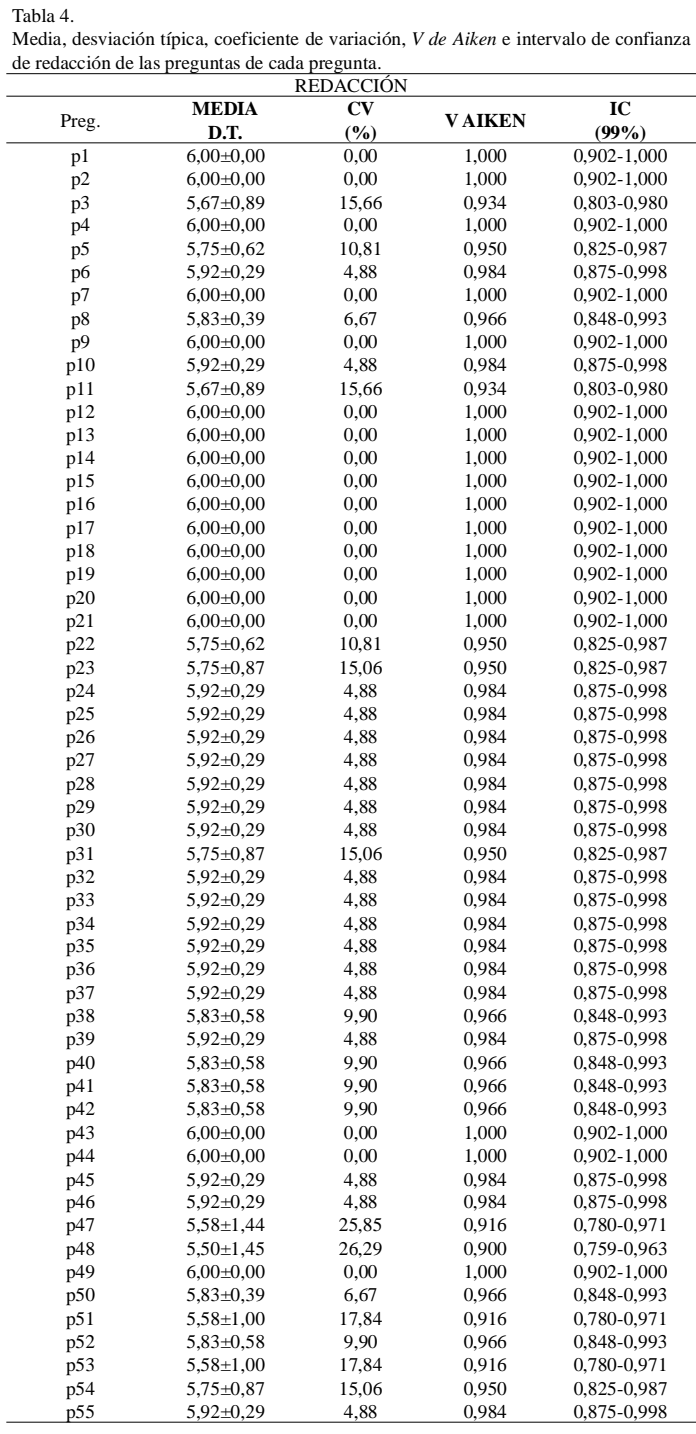

Al final del análisis cualitativo por parte de los expertos se reformularon todos los aspectos de redacción y de organización del cuestionario. Una vez realizados los análisis correspondientes para la validación del cuestionario se procedió al análisis estadístico para determinar la fiabilidad del mismo. La consistencia interna global del cuestionario a través del Alfa de Cronbach fue de 0.915. La fiabilidad testretest, resultó en un coeficiente de correlación intraclase de 0.890 $(p<0.01)$.

\section{Discusión}

Los resultados obtenidos apoyan la validez y fiabilidad del cuestionario como instrumento adecuado para el estudio de la formación de los entrenadores de fútbol base. En este sentido, reforzamos la utilización del cuestionario como instrumento de investigación, otorgando una excelente herramienta para que podamos investigar sobre el entrenador (Ortega et al., 2008). Por otra parte, como han señalado Thomas y Nelson (2007), el cuestionario también resultó un instrumento de fácil aplicación en la muestra piloto para el análisis de fiabilidad y que conllevó, a su vez, cerca de 20 minutos de respuesta, lo que representa una pérdida de poco tiempo para la recogida de información.

En la validación de contenido del cuestionario con un análisis cuantitativo y cualitativo fue importante adecuar el perfil de los juecesexpertos a la materia de estudio, como refiere Juan-Llamas (2015). Participó un grupo multidisciplionar de 12 sujetos, siendo un número aceptable para la validación del instrumento según Ortega et al. (2008).

El proceso de validación, en una primera fase general para evaluar la

\begin{tabular}{|c|c|c|c|c|}
\hline p56 & $6,00 \pm 0,00$ & 0,00 & 1,000 & $0,902-1,000$ \\
\hline p57 & $5,83 \pm 0,58$ & 9,90 & 0,966 & $0,848-0,993$ \\
\hline p58 & $5,92 \pm 0,29$ & 4,88 & 0,984 & $0,875-0,998$ \\
\hline p59 & $5,83 \pm 0,39$ & 6,67 & 0,966 & $0,848-0,993$ \\
\hline p60 & $5,75 \pm 0,87$ & 15,06 & 0,950 & $0,825-0,987$ \\
\hline p61 & $5,75 \pm 0,87$ & 15,06 & 0,950 & $0,825-0,987$ \\
\hline p62 & $6,00 \pm 0,00$ & 0,00 & 1,000 & $0,902-1,000$ \\
\hline p63 & $5,42 \pm 1,00$ & 18,39 & 0,884 & $0,740-0,953$ \\
\hline p64 & $5,92 \pm 0,29$ & 4,88 & 0,984 & $0,875-0,998$ \\
\hline p65 & $6,00 \pm 0,00$ & 0,00 & 1,000 & $0,902-1,000$ \\
\hline p66 & $5,67 \pm 1,15$ & 20,38 & 0,934 & $0,803-0,980$ \\
\hline p67 & $5,67 \pm 1,15$ & 20,38 & 0,934 & $0,803-0,980$ \\
\hline p68 & $6,00 \pm 0,00$ & 0,00 & 1,000 & $0,902-1,000$ \\
\hline p69 & $5,92 \pm 0,29$ & 4,88 & 0,984 & 0,875-0,998 \\
\hline p70 & $6,00 \pm 0,00$ & 0,00 & 1,000 & $0,902-1,000$ \\
\hline p71 & $5,92 \pm 0,29$ & 4,88 & 0,984 & 0,875-0,998 \\
\hline p72 & $5,50 \pm 1,45$ & 26,29 & 0,900 & $0,759-0,963$ \\
\hline p73 & $6,00 \pm 0,00$ & 0,00 & 1,000 & $0,902-1,000$ \\
\hline p74 & $5,92 \pm 0,29$ & 4,88 & 0,984 & $0,875-0,998$ \\
\hline p75 & $5,92 \pm 0,29$ & 4,88 & 0,984 & $0,875-0,998$ \\
\hline p76 & $6,00 \pm 0,00$ & 0,00 & 1,000 & $0,902-1,000$ \\
\hline p77 & $5,92 \pm 0,29$ & 4,88 & 0,984 & $0,875-0,998$ \\
\hline p78 & $5,92 \pm 0,29$ & 4,88 & 0,984 & $0,875-0,998$ \\
\hline p79 & $5,83 \pm 0,58$ & 9,90 & 0,966 & $0,848-0,993$ \\
\hline $\mathrm{p} 80$ & $6,00 \pm 0,00$ & 0,00 & 1,000 & $0,902-1,000$ \\
\hline p81 & $6,00 \pm 0,00$ & 0,00 & 1,000 & $0,902-1,000$ \\
\hline p82 & $5,92 \pm 0,29$ & 4,88 & 0,984 & $0,875-0,998$ \\
\hline p83 & $5,58 \pm 1,44$ & 25,85 & 0,916 & $0,780-0,971$ \\
\hline $\mathrm{p} 84$ & $5,67 \pm 1,15$ & 20,38 & 0,934 & $0,803-0,980$ \\
\hline p85 & $5,58 \pm 1,44$ & 25,85 & 0,916 & $0,780-0,971$ \\
\hline p86 & $5,92 \pm 0,29$ & 4,88 & 0,984 & $0,875-0,998$ \\
\hline p87 & $5,83 \pm 0,58$ & 9,90 & 0,966 & 0,848-0,993 \\
\hline p88 & $6,00 \pm 0,00$ & 0,00 & 1,000 & $0,902-1,000$ \\
\hline p89 & $6,00 \pm 0,00$ & 0,00 & 1,000 & $0,902-1,000$ \\
\hline p90 & $5,92 \pm 0,29$ & 4,88 & 0,984 & $0,875-0,998$ \\
\hline p91 & $5,92 \pm 0,29$ & 4,88 & 0,984 & 0,875-0,998 \\
\hline p92 & $5,83 \pm 0,58$ & 9,90 & 0,966 & $0,848-0,993$ \\
\hline p93 & $6,00 \pm 0,00$ & 0,00 & 1,000 & $0,902-1,000$ \\
\hline p94 & $6,00 \pm 0,00$ & 0,00 & 1,000 & $0,902-1,000$ \\
\hline p95 & $6,00 \pm 0,00$ & 0,00 & 1,000 & $0,902-1,000$ \\
\hline p96 & $5,83 \pm 0,58$ & 9,90 & 0,966 & 0,848-0,993 \\
\hline p97 & $6,00 \pm 0,00$ & 0,00 & 1,000 & $0,902-1,000$ \\
\hline p98 & $5,92 \pm 0,29$ & 4,88 & 0,984 & 0,875-0,998 \\
\hline p99 & $5,92 \pm 0,29$ & 4,88 & 0,984 & $0,875-0,998$ \\
\hline p100 & $6,00 \pm 0,00$ & 0,00 & 1,000 & $0,902-1,000$ \\
\hline p101 & $6,00 \pm 0,00$ & 0,00 & 1,000 & $0,902-1,000$ \\
\hline p102 & $5,92 \pm 0,29$ & 4,88 & 0,984 & 0,875-0,998 \\
\hline p103 & $6,00 \pm 0,00$ & 0,00 & 1,000 & $0,902-1,000$ \\
\hline p104 & $5,83 \pm 0,58$ & 9,90 & 0,966 & 0,848-0,993 \\
\hline p105 & $6,00 \pm 0,00$ & 0,00 & 1,000 & $0,902-1,000$ \\
\hline p106 & $5,58 \pm 1,16$ & 20,86 & 0,916 & $0,780-0,971$ \\
\hline p107 & $6,00 \pm 0,00$ & 0,00 & 1,000 & $0,902-1,000$ \\
\hline p108 & $6,00 \pm 0,00$ & 0,00 & 1,000 & $0,902-1,000$ \\
\hline p109 & $5,92 \pm 0,29$ & 4,88 & 0,984 & 0,875-0,998 \\
\hline p110 & $5,92 \pm 0,29$ & 4,88 & 0,984 & $0,875-0,998$ \\
\hline p111 & $6,00 \pm 0,00$ & 0,00 & 1,000 & $0,902-1,000$ \\
\hline p112 & $6,00 \pm 0,00$ & 0,00 & 1,000 & $0,902-1,000$ \\
\hline p113 & $5,75 \pm 0,87$ & 15,06 & 0,950 & $0,825-0,987$ \\
\hline p114 & $5,75 \pm 0,87$ & 15,06 & 0,950 & 0,825-0,987 \\
\hline p115 & $5,75 \pm 0,87$ & 15,06 & 0,950 & 0,825-0,987 \\
\hline p116 & $5,83 \pm 0,58$ & 9,90 & 0,966 & $0,848-0,993$ \\
\hline p117 & $5,92 \pm 0,29$ & 4,88 & 0,984 & 0,875-0,998 \\
\hline
\end{tabular}

presentación, instrucciones, dificultad, orden y extensión del cuestionario, resultó muy adecuado para cuantificar aspectos cualitativos de la herramienta elaborada (Sierra, 2001). En esta validación general sólo la extensión de cuestionario obtuvo una puntuación menos positiva ( $V$ Aiken de 0.5) hecho que se tuvo en cuenta intentando recabar toda la información pertienente. En la segunda fase, más específica, para validar primero cada dimensión del cuestionario, todas las dimensiones obtuvieron valores de $V$ Aiken muy positivos en todas las categorías de valoración: pertinencia, redacción, claridad, comprensión y plenitud, con valores claramente superiores a los exigidos (Penfield \& Giacobbi, 2004), y atendiendo el valor crítico exacto de la V deAiken para grandes muestras, es decir, ajustando el valor, en este caso, a las condiciones del cuestionario, aspecto que tambien tienen en cuenta González-Espinosa, Ibáñez, Feu, y Galatti (2017), en su estudio para la validación de un programa de intervención.

Porúltimo, en la tercera fase de validación se llevó a cabo un análisis más profundo, donde se analizó individualmente cada uno de los 117 ítems del cuestionario (Abad et al., 2011). Con estos tres niveles de validación se pretendió garantizar que el cuestionario registre efectivamente aquello que pretende medir, buscando que tanto el diseño como su validación tengan una fuerte rigurosidad científica (Ortega et al., 2008).

Los resultados obtenidos del análisis cuantitativo muestran que todas las preguntas alcanzaron un coeficiente $V$ de Aiken por encima del valor crítico establecido 0.71. Mostraron, además, valores dentro del intervalo de confianza teniendo en cuenta un margen de error con un nivel del 99\%, lo que nos permite comprobar que la magnitud obtenida 
del coeficiente Vde Aiken fue superior a la establecida como mínimamente aceptable (Merino \& Livia, 2009). A pesar de que cada pregunta obtuvo valores aceptables respecto al promedio (Bulger \& Hourner, 2007), solamente sieteítems mostraron un coeficiente de variación ligeramente superior al establecido (25\%). No obstante, cuando se aplicó el primer criterio de exclusión no se eliminó ninguna pregunta. Por otra parte, se detectaron nueve ítems que mostraron un coeficiente de variación superior al 20\% en el parámetro de redacción, por lo que fueron revisados, criterio también contemplado en otros estudios sobre validación de un instrumento de medida (Gómez-Carmona, Cervera \& Benito, 2014).

Las aportaciones realizadas por los jueces-expertos son indispensables en el desarrollo de un instrumento (Bulger \& Housner, 2007), además de considerarse un elemento esencial para proporcionar evidencias teóricas de validez (Rubio, Berg-Weger, Tebb, Lee, \& Rauch, 2003). Estas aportaciones permitieron ajustar tanto el contenido como la redacción de las preguntas (Ortega et al., 2008). La gran mayoría de las aportaciones realizadas se centraron en aspectos morfosintácticos, lo que permitió definir cuidadosamente la pregunta que se pretendía evaluar (Osterlind, 1989). Una de las aportaciones más importante hacía referencia a señalar directamente en el cuestionario las dimensiones del mismo, como se hizo en otras investigaciones (Ortega et al., 2018). Esta pertinente sugerencia hizo que se reorganizara el orden y nuremación del cuestionario definitivo. Por otra parte, los expertos sugirieron la incorporación de nuevas preguntas al cuestionario, lo que ya había ocurrido en otros estudios similares (Robles et al., 2016).

Teniendo en cuenta ambos análisis cuantitativo y cualitativo, se han revisado todos los ítems modificándose 23 de ellos. El cuestionario preliminar estaba constituido por 117 ítems, tras los pertinentes cambios quedó finalmente formado por 121 ítems estructurados del siguiente modo: dimensión Caracteristicas Sociodemograficas (5ítems), dimensión Experiencia Deportiva (6 ítems), dimensión Aspectos Personales (12 ítems), dimensión Formación como Entrenador (21 ítems), dimensión Formación Continua (9 ítems), dimensión Concepción de Enseñanza (10ítems), dimensión Planificación eProgramación(20ítems) y dimensión Necessidades de Formación (38 ítems).

El cuestionario definitivo mostró tener una excelente consistencia interna global (alpha = 0.915), considerando el cuestionario, de medición excelente (George \& Mallery, 1995). Los resultados en el coeficiente de correlación intraclase (CCI 0.890) muestran una alta fiabilidad test-retest (Butragueño \& Benito, 2014).

\section{Conclusiones}

El objetivo de este trabajo fue diseñar y validar un cuestionario para su aplicación en los clubes de la Asociación de Fútbol de Beja(Portugal) En este sentido, se ha obtenido un instrumento que muestra indicadores psicométricos suficientes para ser considerado válido y fiable para ser utilizado en el estudio y análisis de la formación de los entrenadores. Con el diseño y la validación de este cuestionario, los investigadores y los responsables de la formación de los entrenadores, a partir de ahora, cuentan con un instrumento útil y práctico para estudiar la formación de entrenadores de fútbol base.

Por otra parte, consideramos como puntos fuertes, el haber contado para la validación del cuestionario con un grupo multidisciplinar con consolidada experiencia en el ámbito de la investigación y del entrenamiento deportivo. La principal dificultad encontrada en el proceso llevado a cabo se relaciona con el momento del retest del cuestionario en relación con las condiciones y disponibilidad de respuesta para que sean las mismas en comparación con el primer momento de contacto con el cuestionario.

Creemos que en futuras investigaciones el presente cuestionario podrá ser utilizado para estudiar el panorama de los entrenadores en todo Portugal con el fin de conocer los aspectos que caracterizan a los entrenadores de jóvenes futbolistas y su intervención. Para ello es imprescindible contar con instrumentos que permitan entender y analizar mejor la formación del entrenador de formación.

\section{Referencias}

Abad, M. T., Benito, P. J., Giménez, F. J., \& Robles, J. (2011). La formación de los entrenadores de fútbol base en la provincia de Huelva. Cultura Ciencia e Deporte, 6(18), 171-183.

Abad, M. T., Giménez, F. J., Robles, J., \& Castillo, E. (2013). La formación de los entrenadores de jóvenes futbolistas. Ebalonmano.com: Revista de Ciencias del Deporte 9(2), 105-114.

Abad, M. T., Giménez, F. J., Robles, J., \& Rodríguez, J. M. (2011). Perfil, experiencia y métodos de enseñanza de los entrenadores de jóvenes futbolistas en la provincia de Huelva. Retos. Nuevas tendencias en Educación Física, Deporte y Recreación, 20, 21-25.

Aiken, L. R. (1985). Three coefficients for analyzing the reliability and validity of ratings. Educational and Psychological Measurement, 45, 131-142.

Almonacid-Fierro, A., Feu, S., \& Vizuete, M. (2018). Validación de un cuestionario para medir el Conocimiento Didáctico del Contenido en el profesorado de Educación Física. Retos. Nuevas tendencias en Educación Física, Deportey Recreación, 34, 132-137.

Azpillaga, I., González, Ó., Irazusta, S., \& Arruza, J.A. (2012). Análisis y valoración de la influencia que ejerce el perfil formativo de los entrenadores en jóvenes futbolistas. Retos. Nuevas tendencias en Educación Física, Deporte y Recreación, 22, 62-64.

Bettega, O., Scaglia, A., Nascimento, J., Ibáñez, S., \& Galatti, L. (2018). O ensino da tática e da técnica no futebol: concepção de treinadores das categorias de base. Retos. Nuevas tendencias en Educación Física, Deporte y Recreación, 33, 112-117.

Bulger, S. M., \& Hourner, L. D. (2007). Modified delphi investigation of exercise science in physical education teacher education. Journal of Teaching in Physical Education, 26, 57-80.

Burgos, R. (2006). Metodología de investigación y escritura científica en clínica. Granada: Escuela Andaluza de Salud Pública.

Butragueño, J., \& Benito, P. J. (2014). Validation of an instrument for injury data collection in strength training. European Journal of Human Movement, 33, 152-167.

Castañer, M., Camerino, O., \& Anguera, M. T. (2013). Métodos mixtos en la investigación de las ciencias de la actividad física y el deporte. Apunts. Educación Física y Deportes, 112(2), 31-36. doi:10.5672/apunts.2014-0983.es.(2013/2).112.01

Charter, R. A. (2003). A breakdown of reliability coefficients by test type and reliability method, and the clinical implications of low reliability. Journal of General Psychology, 130(3), 290-304.

Checa, I. \& Bohórquez, M.R. (2018). Validación española del cuestionario de locus de control en deporte infantil / Spanish Validation of the Locus of Control Questionnaire in Sport for Children. Revista Internacional de Medicina y Ciencias de la Actividad Física y el Deporte, 18(71). doi:http://dx.doi.org/10.15366/rimcafd2018.71.011

Cunha, G. B., Mesquita, I. M. R., Rosado, A. F. B., Sousa, T., \& Pereira, P. (2010). Necessidades de formação para o exercício profissional na perspectiva do treinador de Futebol em função da sua experiência enível de formação. Motriz, 16(4), 31-41.

García-Martín, A., Antúnez, A., \& Ibáñez, S. J. (2016). Análisis del proceso formativo en jugadores expertos: validación de instrumento. Revista Internacional de Medicina y Ciencias de la Actividad Físicayel Deporte,16(61), 157-182. doi:http://dx.doi.org/10.15366/ rimcafd2016.61.012

Garrido, M. E., Zagalaz, M. L., Torres G., \& Romero, S. (2010). Diseño y validación de un cuestionario para técnicos deportivos acerca de su opinión sobre las actitudes de padres y madres en el deporte (CTPMD). Cuadernos de Psicología del Deporte, 10(2), 7-21.

George, D., \& Mallery, P. (1995). SPSS/PC+ step by step: Asimple guide and reference. Belmont(Estados Unidos): Wadsworth Publishing Company.

Giménez, F. J., Abad, M. T., \& Robles, J. (2010). El proceso de formación del jugador durante la etapa de iniciación deportiva. Apunts. Educación Física y Deportes, 99, 47-55. 
Gómez-Carmona, P. M., Cervera, V., \& Benito, P. J. (2014). Diseño y validación de un cuestionario socio-emocional para jóvenes futbolistas de élite. Revista Internacional de Medicinay Ciencias de la Actividad Física y el Deporte, 14(55), 545-559.

González-Espinosa, S., Ibáñez, S. J., Feu, S., \& Galatti, L. R. (2017). Programas de intervención para la enseñanza deportiva en el contexto escolar, PETB y PEAB. Retos. Nuevas Tendencias en Educación Física, Deporte y Recreación, 31, 103-106.

Juan-Llamas, C. (2015). Diseño y validación de un cuestionario sobre la forma de trabajo de los instructores de clases colectivas. Retos. Nuevas Tendencias en Educación Física, Deporte y Recreación, 27, 19-23.

Lago, C. (2009). The influence of match location, quality of opposition, and match status on possession strategies in professional association football. Journal of Sports Sciences, 27(13), 1463-1469.

Latorre, P. A., \& Pantoja, A. (2012). Diseño y validación de una escala de percepción del riesgo en actividades físicodeportivas Escolares. Retos. Nuevas tendencias en Educación Física, Deporte y Recreación, 21, 25-29.

Light, R. L., \& Harvey, S. (2017). Positive Pedagogy for sport coaching. Sport, Education and Society, 22(2), 271-287. doi:10.1080/ 13573322.2015.1015977

Lledó, E., Martínez, G, \& Huertas, F. (2014). Perfil del entrenador de fútbol en la etapa escolar en escuelas de clubes deélite de la Comunitat Valenciana. Cultura Ciencia y Deporte, 9(25), 57-68.

Maldonado, E., Pacheco, R., \& Zamarripa, J. (2017). Validación mexicana del cuestionario de clima de aprendizaje adaptado a la educación física. Retos. Nuevas Perspectivas de Educación Física, Deportey Recreación, 32, 115-118.

Merino, C., \& Livia, J. (2009). Intervalos de confianza asimétricos para el índice la validez de contenido: Un programa Visual Basic para la V de Aiken. Anales de psicología, 25(1), 169-171.

Mesquita, I., Isidro, S., \& Rosado, A. (2010). Portuguese coaches' perceptions of and preferences for knowledge sources related to their professional background. Journal of Sports Science and Medicine, 9(3), 480-489.

Nevil, A. M., Lane, A. M., Kilgour, L. J., Bowes, N., \& Whyte, G. P. (2001). Stability of psychometric questionnaires. Journal of Sports Sciences, 19(4), 273-278.

Nuviala, A., Tamayo, J. A., Iranzo, J., \& Falcón, D. (2008). Creación, diseño, validación y puesta en práctica de un instrumento de medición de la satisfacción de usuarios de organizaciones que prestan servicios deportivos. Retos. Nuevas tendencias en Educación Física, Deporte y Recreación, 14, 10-16.

Ortega, E., Calderón, A., Palao, J. M., \& Puigcerver, M. C. (2008). Diseño y validación de un cuestionario para evaluar la actitud percibida del profesor en clase y de un cuestionario para evaluar los contenidos actitudinales de los alumnos durante las clases de educación física en secundaria. Retos. Nuevas tendencias en Educación Física, Deporte y Recreación, 14, 22-29.

Ortega, G., Abad, M. T., Giménez, F. J., Durán, L. J., Franco, J., Jiménez, A. C., \& Robles, J. (2018). Diseño y validación de un cuestionario de satisfacción con programas deportivos en centros penitenciarios. Apunts. Educación Física y Deportes, 131(1), 21-33. doi:10.5672/apunts.2014-0983.es.(2018/1).131.02

Osterlind, S. J. (1989). Constructing Test Items. Londres: Kluwer Academic Publishers.

Östlund, U., Kidd, L., Wengström, Y., \& Rowa-Dewar, N. (2011). Combining qualitative and quantitative research within mixed method research designs: A methodological review. International Journal of Nursing Studies, 48, 369-383. doi:10.1016/ j.jjnurstu.2010.10.005

Otero, F. M., González, J. A., \& Calvo, A. (2012). Validación de instrumentos para la medición del conocimiento declarativo y procedimental y la toma de decisiones en el fútbol escolar. Retos. Nuevas tendencias en Educación Física, Deporte y Recreación, 22(2), 65-69.
Penfield, R. D., \& Giacobbi, P. R. (2004). Appying ascore confidence interval to Aiken's item contentrelevance index. Measurement in Physical Education and Exercise Science, 8(4), 213-225. doi:10.1207/ s15327841mpee0804_3

Pereira, A. (2008). SPSS Guia Prático de Utilização, Análise de dados para ciências sociais e psicologia. Lisboa. Edições Sílabo, Lda.

Pulido, J. J., Sánchez-Oliva, D., Sánchez-Miguel, P. A., Leo, F. M., \& García-Calvo, T. (2016). Influencia de la formación de los entrenadores sobre la motivación de los deportistas. Revista Internacional de Medicina y Ciencias de la Actividad Física yel Deporte, 16(64), 685-702. doi:10.15366/rimcafd2016.64.005

Robles, A., Robles, J., Giménez, F. J. \& Abad, M. T. (2016). Validación de una entrevista para estudiar el proceso formativo de judokas de élite. Revista Internacional de Medicina y Ciencias de la Actividad Física y el Deporte, 16(64), 723-738. doi:10.15366/ rimcafd2016.64.007

Rubio, D. M., Berg-Weger, M., Tebb, S. S., Lee, E. S., \& Rauch, S. (2003). Objectifying content validity: Conducting a content validity study in social work research. Social Work Research, 27, 94-104.

Saldaña, L. M. \& García, J. M. (2013). Diseño, creación y validación de una entrevista para obtener datos biográficos, de carácter deportivo-militar, de los militares que participaron en unos juegos olímpicos. Journal of Sport and Health Research, 5(2),157-166.

Sierra, B. R. (2001). Técnicas de Investigación Social: teoría y ejercicios (Decimocuarta ed.). Madrid: Paraninfo.

Simón, J. A., Fernández, J. G, \& Contreras, O. R. (2017). Diseño y validación de un cuestionario de autopercepción de la excelencia en el deporte Design and validation of a questionnaire on self-perception of excellence in sport. Retos. Nuevas tendencias en Educación Física, Deporte y Recreación, 31, 58-63.

Stoszkowski, J. \& Collins, D. (2017). Using shared online blogs to structure and support informal coach learning - part 1: a tool to promote reflection and communities of practice. Sport, Education and Society, 22(2), 247-270. doi:10.1080/13573322.2015.1019447

Taylor, W. G., Piper, H., \& Garratt, D. (2016). Sports coaches as 'dangerous individuals'-practice as governmentality. Sport, Education and Society, 21(2), 183-199. doi:10.1080/ 13573322.2014.899492

Thomas, J. R., \& Nelson, J. K. (2007). Métodos de investigación en actividad física. Barcelona: Paidotribo.

Yagüe, J. M., Herrero, A., Tabernero, B., \& Veroz, R. (2017). Diseño y validación del cuestionario «Deporteduca» para conocer la incidencia de la competición sobre algunos valores socioeducativos en el ámbito de la iniciación al fútbol. Retos. Nuevas tendencias en Educación Física, Deporte y Recreación, 31, 197-201.

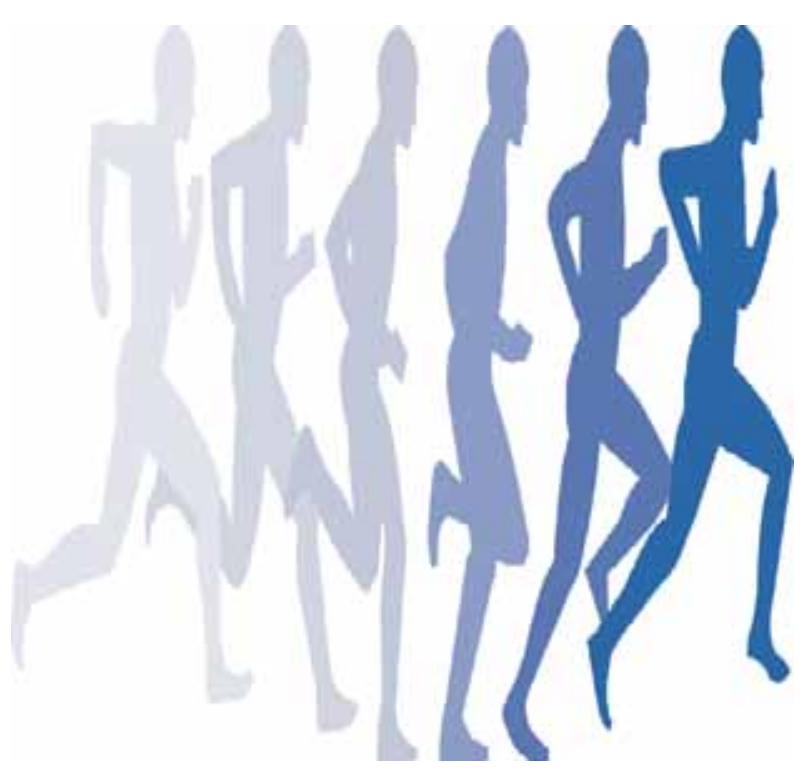

